\title{
Avaliação do consumo de corantes artificiais por lactentes, pré-escolares e escolares
}

\author{
Evaluation of artificial dye intake by children
}

\author{
Simone Pinheiro Alves SCHUMANN ${ }^{1}$, Maria Lucia Teixeira POLÔNIO ${ }^{1}$, \\ Édira Castello Branco de Andrade GONÇALVES ${ }^{1 *}$
}

\section{Resumo}

O objetivo do presente trabalho foi avaliar o consumo, por 150 crianças de até 10 anos, de pó para gelatina, preparado sólido para refresco e refrigerante. Foi feita aplicação de questionário de freqüência quantitativa e qualitativa com população infantil atendida no ambulatório de pediatria do Hospital Universitário Gafrée Guinle, Rio de Janeiro. Os dados foram tratados estatisticamente pelo programa SPSS 8.0. Verificou-se que os alimentos pesquisados são ampla e freqüentemente consumidos, sendo introduzidos na dieta, em muitos dos casos, antes da criança completar 1 ano de idade. Sabe-se que os produtos estudados não são adequados nutricionalmente, pois apresentam, além dos corantes, outros aditivos alimentares e não contêm nenhum nutriente importante para o funcionamento do organismo. Em relação aos corantes presentes nos produtos mencionados pela população estudada, constatou-se que os mais informados na rotulagem destes produtos, no item ingredientes, são amarelo crepúsculo, tartrazina e amaranto. Estimando que o teor dos corantes nos alimentos analisados esteja no limite máximo permitido pela legislação, observou-se que a grande maioria das crianças avaliadas pode estar excedendo a ingestão diária aceitável para o corante amaranto e que cerca de $20 \%$ da população pode estar excedendo o consumo recomendado para o amarelo crepúsculo. É importante que os valores máximos permitidos de corantes em alimentos, no Brasil, sejam adequados às recomendações realizadas pelo órgão internacional que avalia a segurança dos aditivos, e que ações educativas sejam realizadas.

Palavras-chave: crianças; gelatina; refresco; refrigerante; corantes; legislação.

\begin{abstract}
This study aimed at evaluating the intake of solid jelly powder, solid juice powder and soft drinks by children. The intakes were estimated through the application of a quantitative and qualitative food frequency questionnaire. The study was pursued in the pediatric nutrition ambulatory of Gaffrée Guinle University Hospital in Rio de Janeiro, Brazil. Data were treated with the SPSS 8.0 software in order to analyze the frequencies and crossing of variables. It was verified that children of different ages consumed variable amounts of artificial colorings through widespread industrialized products, introduced, in many cases, before 1 year of age. The industrialized products mentioned by the surveyed population declared in their labels to contain artificial colorings such as sunset yellow (FD\&C Yellow no. 6), tartrazine (FD\&C Yellow no. 5) and amaranth (FD\&C Red no. 2). The individual contributions of the coloring additives to the daily intake of the subjects were added in order to compare with the maximum levels allowed by the current regulation. It was observed that the great majority of the children evaluated surpassed the daily ingestion permitted for amaranth; $20 \%$ of the children surpassed the daily ingestion for sunset yellow. Compliance with the JECFA's recommendations and the Brazilian legislation shall only be attained by promoting educational actions that warn about the risks and stimulate the rational use of these products by consumers.
\end{abstract}

Keywords: children; solid jelly powder; solid juice powder; soft drinks; legislation.

\section{Introdução}

Os aditivos alimentares têm sido cada vez mais utilizados no desenvolvimento de novos produtos, com o objetivo de melhorar aparência, aroma, sabor, cor, textura, valor nutritivo e conservação. Ao considerar que o aspecto visual é um fator importante para a seleção e escolha do produto, os corantes destacam-se entre uma das classes de aditivos imprescindíveis para a indústria alimentícia na conquista de mercados (ASHFAQ; MASUD, 2002; CLYDESDALE, 1993; EVANGELISTA, 2001).

O emprego de corantes em alimentos é motivo de muita polêmica, na medida em que a principal justificativa, em muitos dos casos, é tornar o produto mais atrativo esteticamente. Além disso, estudos vêm demonstrando a ocorrência de reações adversas a curto e longo prazo, devido ao consumo de alimentos que apresentam esses aditivos (BESELER, 1999; KRAUSE; MAHAN, 1998; ORTOLANI et al., 1999).

No que concerne às reações adversas aos aditivos, sabe-se que a população infantil constitui o grupo mais vulnerável. Isto ocorre devido à quantidade ingerida ser maior, em relação ao peso corporal, na criança do que no adulto. Além disso, as crianças encontram-se em um período de alto metabolismo e desenvolvimento de suas defesas naturais e não apresentam

Recebido para publicação em 8/2/2007

Aceito para publicação em 19/10/2007 (002285)

${ }^{1}$ Departamento de Tecnologia de Alimentos, Escola de Nutrição, Universidade Federal do Estado do Rio de Janeiro - UNIRIO, Av. Pasteur, 296, CEP 22290-240,

Praia Vermelha, Rio de Janeiro - RJ, Brasil, E-mail: ediracba@analisedealimentos.com.br

${ }^{*}$ A quem a correspondência deve ser enviada 
capacidade de auto-controle no consumo de alimentos ricos em aditivos (POLÔNIO, 2002; SHILS; OLSON; SHIKE, 2003).

A avaliação segura dos corantes no âmbito mundial é baseada no controle da IDA (Ingestão Diária Aceitável), desenvolvida pelo JECFA (Joint FAO/WHO Committee on Food Additives). No Brasil, a ANVISA (Agência Nacional de Vigilância Sanitária), com a finalidade de minimizar os riscos à saúde humana, publicou em 1999 resoluções que estabeleciam os limites máximos permitidos dos aditivos para as diferentes categorias de alimentos. Contudo, a utilização de aditivos em alimentos suscita uma série de dúvidas como: se as quantidades utilizadas pelas indústrias são adequadas para que o consumo de determinado alimento não permita que seja ultrapassada a IDA, na medida em que não existe obrigatoriedade legal em declarar as quantidades presentes no alimento, mas somente a relação dos aditivos utilizados (BRASIL, 2006a; b; c).

Os estudos de consumo são importantes para estimar o uso seguro dos aditivos alimentares numa determinada população. Um método que pode ser utilizado é o Questionário de Freqüência de Consumo de Alimentos (QFCA), que apresenta como vantagens, o fato de ser um instrumento de baixo custo e fácil de ser aplicado, podendo assim ser utilizado em estudos com grande número de indivíduos. A reprodutibilidade das informações dos questionários de freqüência é razoavelmente boa, e a validade é aceitável quando se faz a comparação da média de ingestão de nutrientes com outros métodos (BEATON; BUREMA; RITENBAUGH, 1994; SALVO; GIMENO, 2002).

Diante do exposto, justifica-se a realização de estudo para levantamento do consumo, por crianças de até 10 anos, de pó para gelatina, preparado sólido para refresco e refrigerante, alimentos coloridos artificialmente amplamente consumidos pela população infantil. Através dos resultados, procurou-se estimar se a quantidade consumida pelas crianças pode ultrapassar a IDA para os corantes mais empregados nos produtos em estudo, considerando os limites máximos preconizados pela legislação para o uso destes aditivos.

\section{Material e métodos}

\subsection{Avaliação de consumo}

Tendo em vista que todos os métodos que avaliam o consumo alimentar são, em algum momento, imperfeitos, e que não existe padrão-ouro em nutrição, o questionário utilizado no estudo foi de freqüência quantitativa e qualitativa sobre o consumo de produtos coloridos artificialmente e habitualmente consumidos por crianças. Esse método foi selecionado pela facilidade de aplicação, baixo custo e por permitir o conhecimento do consumo habitual desses alimentos.

O questionário aplicado encontra-se a seguir:

Questionário de avaliação de consumo alimentar

Nome do responsável:

Nome da criança:

Data de nascimento:

Sexo: ( ) Feminino ( ) Masculino

A criança consome:

( ) Gelatina Idade de introdução:

Caso a criança tenha consumido no mês, responda:

Marca:

Sabor:

Caso não utilize as proporções indicadas pelo fabricante, informe a usada:

Pó: Água:

O consumo foi:

( ) Diário

( ) Semanal

( ) Mensal ( ) Vezes

( ) Refresco em pó Idade de introdução:

Caso a criança tenha consumido no mês, responda:

Marca:

Sabor:

Caso não utilize as proporções indicadas pelo fabricante, informe a usada:

Pó: Água:

O consumo foi:

( ) Diário Quantidade:

( ) Semanal ( ) Vezes Quantidade:

( ) Mensal ( ) Vezes

Quantidade:

( ) Refrigerantes sabor laranja, uva ou citrus

Idade de introdução:

Caso a criança tenha consumido no mês, responda:

Marca:

Sabor:

O consumo foi:
( ) Diário
Quantidade:
( ) Semanal ( ) Vezes
Quantidade:
( ) Mensal
( ) Vezes
Quantidade:

\subsection{Amostra}

A avaliação de consumo foi realizada no ambulatório pediátrico do Hospital Universitário Gafrée Guinle, Rio de Janeiro, com os responsáveis por 150 crianças das seguintes faixas etárias lactentes $(\mathrm{N}=50)(0$ a 1 ano e 11 meses $)$, préescolares $(\mathrm{N}=50)$ ( 2 a 5 anos e 11 meses) e escolares $(\mathrm{N}=50)$ (6 a 10 anos). Não foram consideradas crianças que apresentaram modificações na dieta no mês de estudo. Este trabalho foi aprovado pelo Comitê de Ética do mesmo hospital, e todos 
os responsáveis assinaram o termo de consentimento livre e esclarecido.

\subsection{Estimativa da ingestão diária de corantes $e$ comparação com a IDA}

A fim de estimar a ingestão dos corantes, foi utilizado o limite máximo estabelecido pela legislação para os aditivos de cor mais empregados nos alimentos analisados. Para comparar com a IDA, foi utilizado como referência o peso teórico que se encontra no percentil 50 do padrão de referência, segundo a Tabela do NCHS (1977) em relação à idade atual das crianças avaliadas.

\subsection{Tratamento dos dados}

Os dados foram tratados utilizando o programa SPSS versão 8.0 para análise de freqüências e cruzamento de variáveis.

\section{Resultados e discussão}

O consumo de gelatina, refresco em pó e refrigerante pelas crianças atendidas no Ambulatório Pediátrico do Hospital Universitário Gafrée Guinle, Rio de Janeiro, encontra-se na Figura 1. Observa-se que a maioria $(70 \%)$ ingeriu mais de um alimento colorido artificialmente no mês de estudo e que a gelatina foi o mais citado. Somente $7 \%$ da população não apresenta o hábito de consumir os alimentos pesquisados.

A partir da Figura 2 constata-se que a maioria das crianças iniciou o consumo dos três produtos em estudo antes dos 2 anos de idade, sendo que o pó para gelatina foi introduzido até 1 ano em $95 \%$ dos casos. Este dado é preocupante, uma vez que a IDA estabelecida pela JECFA não pode ser aplicada para crianças com idade inferior a 12 meses devido à adaptação do metabolismo e ao fato de que se preconiza a alimentação somente por meio do aleitamento materno. Por isso, é proibida a adição de aditivos às fórmulas infantis, mas como se percebe produtos coloridos artificialmente, que não estão sujeitos à referida normatização, são também consumidos por essas crianças (POLÔNIO, 2002; SHILS; OLSON; SHIKE, 2003).

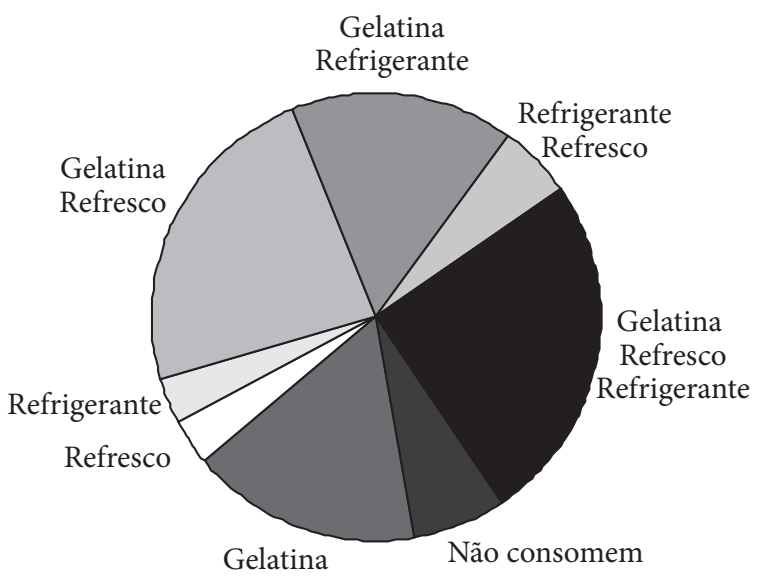

Figura 1. Consumo de produtos alimentícios por crianças de 0 a 10 anos de idade atendidas no Hospital Universitário Gafrée Guinle.
Através da distribuição do consumo por faixa etária, apresentada na Figura 3, verifica-se que as crianças que não ingeriram nenhum dos produtos avaliados no mês de estudo são principalmente lactentes com menos de 1 ano de idade. Além disso, percebe-se que esse é o grupo que apresenta a menor proporção de crianças que consumiram os alimentos estudados. Porém, os dados são referentes somente a 15 crianças, que correspondem a 33\% do grupo de lactentes e 10\% do total da população analisada.

A partir da Figura 4, verifica-se que os lactentes, quando comparados com as crianças de outras faixas etárias, consumi-

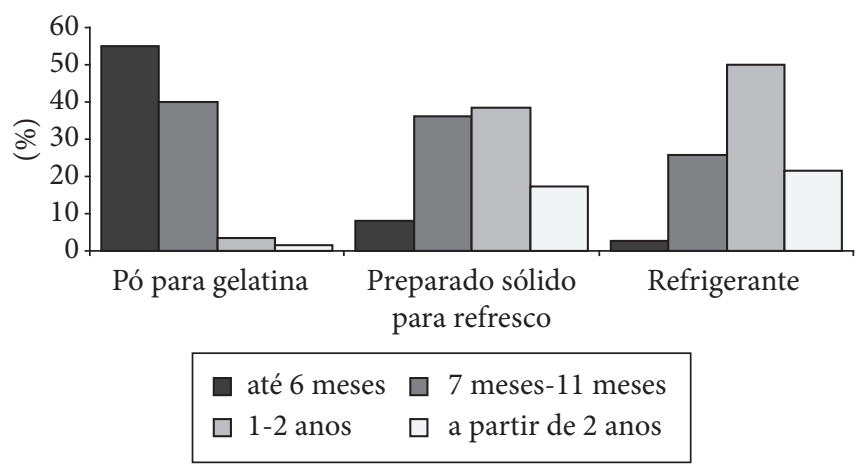

Figura 2. Idade de introdução de produtos alimentícios na dieta de crianças atendidas no Hospital Universitário Gafrée Guinle.

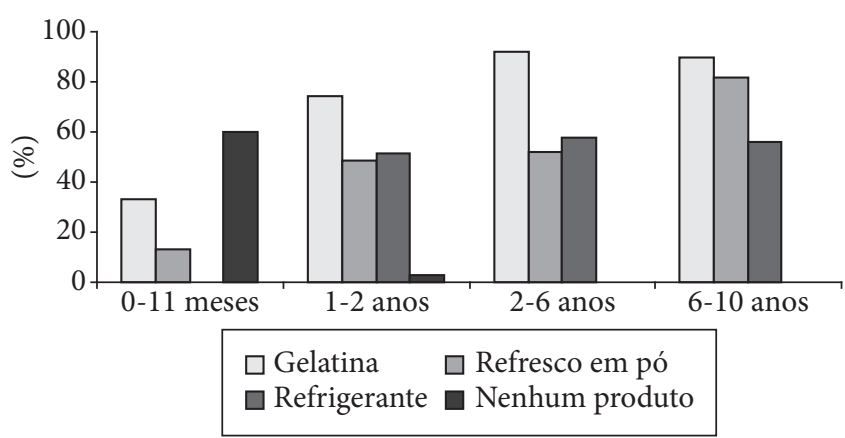

Figura 3. Consumo de produtos alimentícios na faixa etária das crianças atendidas no Hospital Universitário Gafrée Guinle.

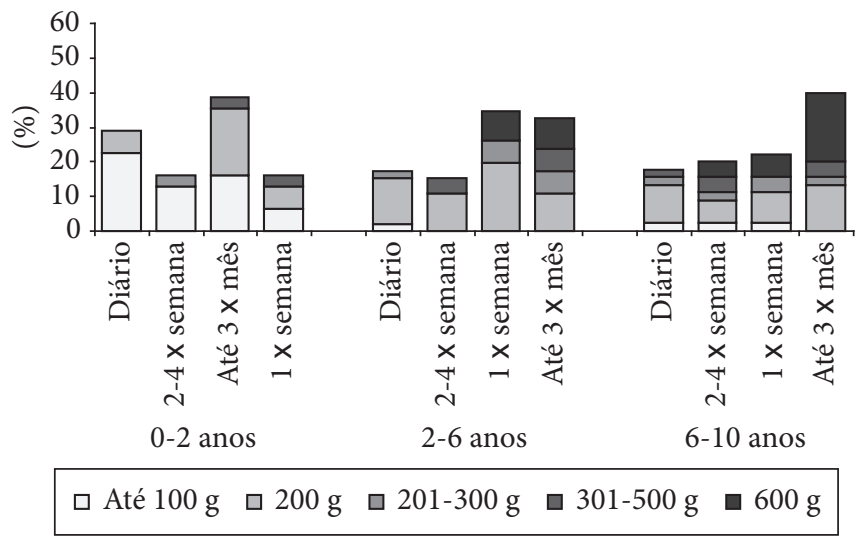

Figura 4. Freqüência quantitativa do consumo de pó para gelatina por crianças de 0 a 10 anos atendidas no Hospital Universitário Gafrée Guinle. 
ram com maior freqüência a gelatina em pó no mês estudado, porém a quantidade ingerida foi menor. Analisando o consumo semanal, constata-se que os pré-escolares foram os maiores consumidores desse produto.

As crianças de 2 a 6 anos são as que ingeriram preparado sólido para refresco mais habitualmente, e os escolares consumiram, semanalmente, cerca de duas vezes mais esse alimento quando comparados às crianças das outras faixas etárias, conforme demonstrado na Figura 5.

Em relação ao consumo de refrigerantes sabores laranja e uva, Figura 6, os lactentes são aqueles que ingeriram mais freqüentemente esse alimento, porém quando se avalia a quantidade consumida por semana, percebe-se que tanto os pré-escolares quanto os escolares ingeriram, aproximadamente, duas vezes mais esse produto.

Constata-se, através da Figura 7, que o corante amarelo crepúsculo é o mais empregado, estando presente em $78 \%$ das amostras, seguido pelos corantes tartrazina e amaranto, que

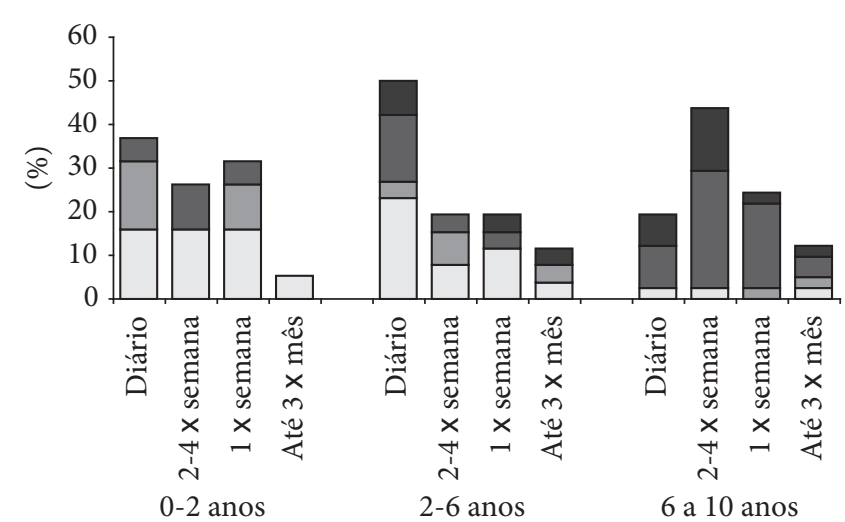

口Até $200 \mathrm{~mL} \square 201-300 \mathrm{~mL} \square 400 \mathrm{~mL}$ acima de $400 \mathrm{~mL}$

Figura 5. Freqüência quantitativa do consumo de preparado sólido para refresco por crianças de 0 a 10 anos atendidas no Hospital Universitário Gafrée Guinle.

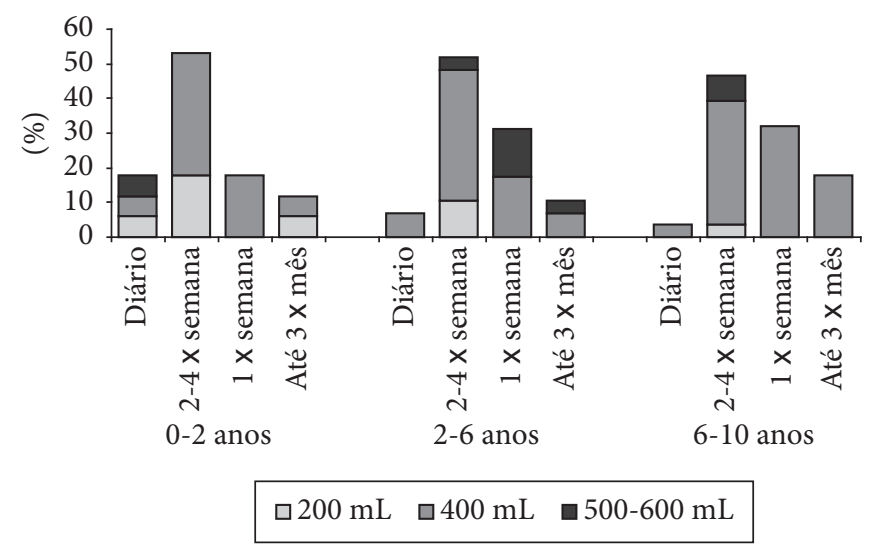

Figura 6. Freqüência quantitativa do consumo de refrigerantes por crianças de 0 a 10 anos atendidas no Hospital Universitário Gafrée Guinle. estão na composição de 57 e 44\% dos alimentos estudados, respectivamente. Ao analisar os diferentes produtos, nota-se que a tartrazina é o corante mais utilizado em preparado sólido para refresco; enquanto o amarelo crepúsculo e o amaranto predominam no pó para gelatina e refrigerante.

Considerando os dados acima, cujos corantes tartrazina, amarelo crepúsculo e amaranto foram os predominantes nos alimentos estudados, foi feita uma comparação do teor máximo permitido pela legislação brasileira, com a IDA determinada pelo JECFA.

Observa-se uma discrepância no que diz respeito à recomendação diária e ao valor permitido dos corantes pela legislação. Para o amaranto, cuja IDA é de até $0,5 \mathrm{mg} \cdot \mathrm{kg}^{-1}$, a legislação permite adição máxima de 10 a 20 vezes à IDA. Já para o amarelo crepúsculo, esta relação ocorre na proporção de 4 vezes e no caso da tartrazina 2 vezes.

Foi feita uma estimativa da ingestão diária dos aditivos amarelo crepúsculo e amaranto, considerando que os alimentos analisados apresentavam o teor máximo destes corantes permitido pela legislação. Assim, a relação do consumo com o peso da criança foi calculada considerando-se o peso teórico que se encontra no percentil 50 do padrão de referência, segundo a Tabela 1 do NCHS, na medida em que o peso das crianças não foi mensurado.

Através da Figura 8, percebe-se que $20 \%$ da população em estudo pode estar excedendo a IDA do corante amarelo crepúsculo. Em relação ao amaranto, nota-se, por meio da Figura 9, que a IDA pode estar sendo ultrapassada por mais de $90 \%$ das crianças.

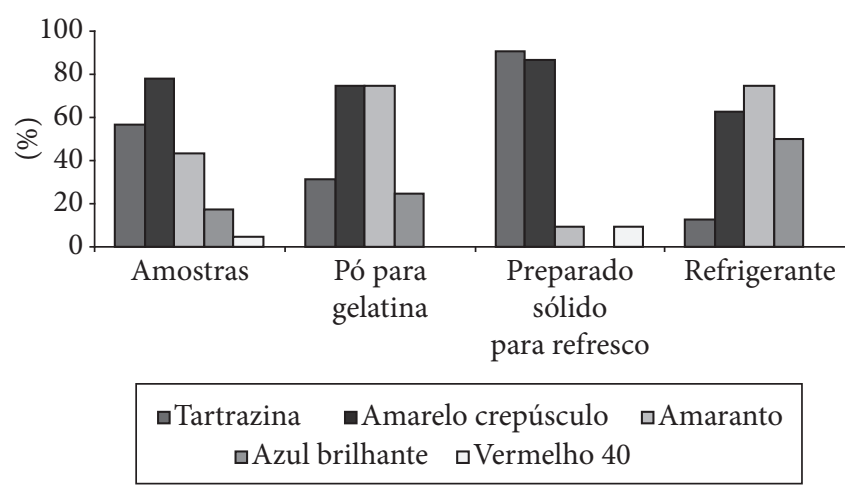

Figura 7. Corantes empregados nas marcas e sabores dos produtos de interesse consumidos por crianças de 0 a 10 anos de idade.

Tabela 1. Limites máximos em produtos alimentícios e IDA para amarelo crepúsculo e amaranto.

\begin{tabular}{lcccc}
\hline Corantes & $\begin{array}{c}\text { Pó para } \\
\text { gelatina } \\
\left(\mathrm{mg} .100 \mathrm{~g} \mathrm{~g}^{-1 \star}\right)\end{array}$ & $\begin{array}{c}\text { Preparado } \\
\text { sólido para } \\
\text { refresco }\end{array}$ & $\begin{array}{c}\text { Refrigerante } \\
\left(\mathrm{mg} .100 \mathrm{~mL}^{-1}\right)\end{array}$ & $\begin{array}{c}\mathrm{IDA}^{3} \\
\left(\mathrm{mg}^{-1} \mathrm{~kg}^{-1}\right)\end{array}$ \\
\hline Amarelo & 10 & $\left.1000 \mathrm{~mL}^{-1 \star}\right)$ & 10 & $0-2,5$ \\
crepúsculo & 10 & 5 & 5 & $0-0,5$ \\
Amaranto & 10 & 10 & 10 & $0-7,5$ \\
Tartrazina & 15 & & & \\
\hline
\end{tabular}

${ }^{*}$ Quantidade do produto pronto para consumo; ${ }^{1}$ resolução n ${ }^{\circ} 388$, de 05 de agosto de 1999 ${ }^{2}$ resolução no 389, de 05 de agosto de 1999; e ${ }^{3} \mathrm{WHO},(2000)$. 


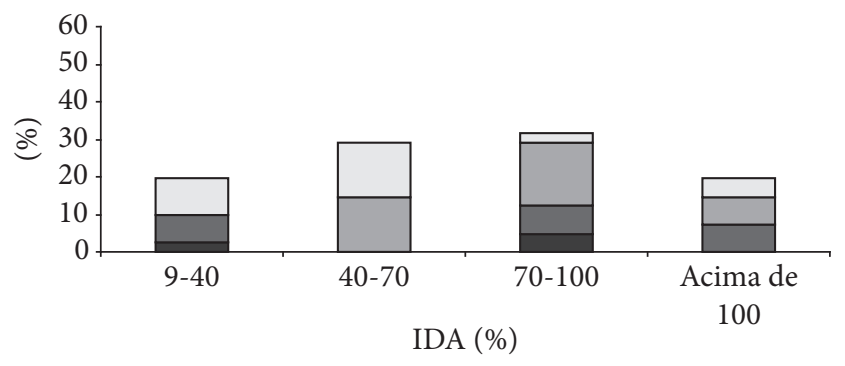

0-1 ano $\square$ 1-2 anos $\square$ 2-6 anos $\square$ 6-10 anos

Figura 8. Contribuição para IDA de consumo de amarelo crepúsculo presente nos produtos analisados segundo faixa etária.

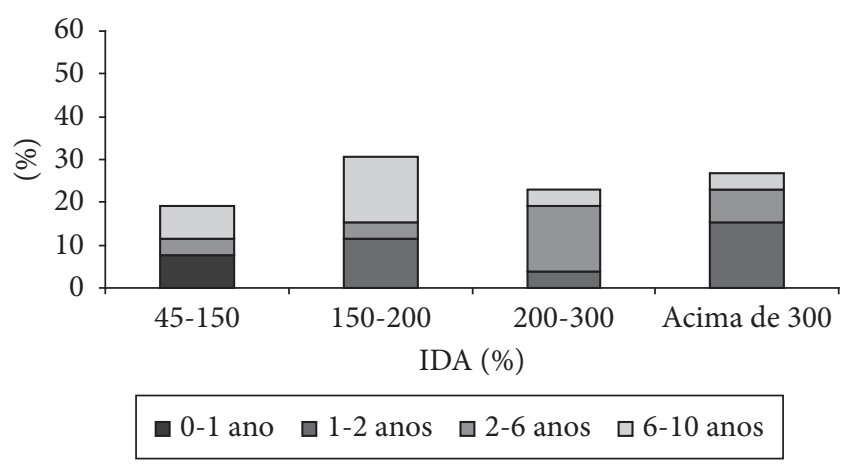

Figura 9. Contribuição para IDA do consumo diário de amaranto presente nos produtos analisados segundo faixa etária.

Mesmo sendo uma estimativa, os dados são preocupantes, principalmente para o corante amaranto, pois foi observado que, para que todas as crianças em estudo não ultrapassassem a IDA, a adição do amaranto nos alimentos em estudo teria que estar nos alimentos com teores inferiores a $25 \%$ do máximo estabelecido pela legislação.

É importante mencionar que este estudo avaliou apenas 3 alimentos que possuem em sua composição estes corantes, mas um número considerável de guloseimas que fazem parte do dia-a-dia das crianças também possui corantes em sua composição. Assim, acredita-se ser de grande valia que os rótulos destes produtos não só mencionem a presença desses corantes, mas que também informem o teor dos mesmos.

\section{Conclusões}

Através do estudo realizado com a população infantil atendida no Ambulatório de Pediatria do Hospital Universitário Gafrée Guinle, Rio de Janeiro, verificou-se que o pó para gelatina e o preparado sólido para refresco e refrigerante são alimentos ampla e freqüentemente consumidos, sendo introduzidos na dieta, muitas vezes, antes da criança completar 1 ano de idade. Sabe-se que esses produtos não são adequados nutricionalmente, pois apresentam, além dos corantes, outros aditivos alimentares.

Em relação aos corantes presentes nos alimentos ingeridos pela população estudada, constatou-se que os mais menciona- dos nos rótulos dos produtos analisados no item ingredientes são: amarelo crepúsculo, tartrazina e amaranto. Ao estimar o consumo destes corantes, considerando a adição dos mesmos no limite máximo preconizado pela legislação, observa-se que a maioria da população em estudo estaria excedendo a IDA para o corante amaranto, e $20 \%$ das crianças estariam ultrapassando a ingestão recomendada para o amarelo crepúsculo.

É importante mencionar que este estudo avaliou apenas três alimentos que possuem em sua composição estes corantes, mas um número considerável de guloseimas que fazem parte do dia-a-dia das crianças também possuem corantes em sua composição. Assim acredita-se ser de grande valia que os rótulos destes produtos não só mencionem a presença desses corantes, mas também informem o teor dos mesmos.

Com isto justifica-se a necessidade de uma vigilância sanitária mais eficaz e eficiente no que diz respeito aos alimentos com aditivos, sendo os limites máximos adequados às recomendações realizadas pelo JECFA. Além disso, campanhas educativas que estimulem o uso racional desses produtos na alimentação infantil devem ser realizadas.

\section{Referências bibliográficas}

ASHFAQ, N.; MASUD, T. Surveillance on Artificial Colours in Different Ready to Eat Foods. Pakistan Journal of Nutrition, v. 1, n. 5, p. 223-225, 2002.

BEATON, G. H.; BUREMA, J.; RITENBAUGH, C. Errors in the interpretation of dietary assessments. American Journal of Clinical Nutrition, v. 65, p. 1100S-1107S, abr. 1997.

BESELER, L. Effects on Behavior and Cognition: Diet and Artificial Colors, Flavors, and Preservatives. International Pediatrics, v. 14, n. 1, p. 41-43, 1999.

BRASIL. Resolução no 386, de 05 de agosto de 1999a. Aprova o Regulamento técnico sobre aditivos utilizados segundo as boas práticas de fabricação e suas funções. Diário Oficial da República Federativa do Brasil, Brasília, DF, 9 de agosto de 1999. Disponível em: <http:// www.anvisa.gov.br>. Acesso em: 21 jul. 2006.

BRASIL. Resolução no 389, de 05 de agosto de 1999b. Aprova o Regulamento técnico que aprova o uso de Aditivos Alimentares, estabelecendo suas Funções e seus Limites Máximos para a Categoria de Alimentos 16: Bebidas - subcategoria 16.2.2 - Bebidas não Alcoólicas Gaseificadas e não Gaseificadas. Diário Oficial da República Federativa do Brasil, Brasília, DF, 9 de agosto de 1999. Disponível em URL: <http:// www.anvisa.gov.br>. Acesso em: 21 jul. 2006.

BRASIL. Leis, decretos, etc... Resolução no 388, de 05 de agosto de 1999c. Aprova o Regulamento técnico que aprova o uso de Aditivos Alimentares, estabelecendo suas Funções e seus Limites Máximos para a Categoria de Alimentos 19 - Sobremesas. Diário Oficial da República Federativa do Brasil, DF, Brasília, 9 de agosto de 1999. Disponível em: <http:// www.anvisa.gov.br>. Acesso em: 21 jul. 2006.

CLYDESDALE, F. M. Color as a factor in food choice. Critical Reviews in Food Science and Nutrition, v. 33, n. 1, p. 83-101, 1993.

EVANGELISTA, J. Tecnologia de Alimentos. São Paulo: Atheneu, 2001.

KRAUSE, S.; MAHAN, L. K. Alimentos, Nutrição e Dietoterapia. São Paulo: Rocca, 1998. 
NCHS - NATIONAL CENTER FOR HEALTH STATISTICS. Growth curves for children birth: 18 years. Washington, DC: Print Office, 1977. (Vital Health Statistics Series, v. 11, n. 165).

ORTOLANI, C. et al. Controversial aspects of adverse reactions to food. Allergy, v. 54, p. 27-45, 1999.

POLÔNIO, M. L. T. Aditivos alimentares e saúde infantil. In: ACCIOLY, E.; SAUNDERS, C.; LACERDA, E. M. Nutrição em Obstetrícia e Pediatria. Rio de Janeiro: Cultura Médica, 2002. p. 511- 527.
SALVO, V. L. M. A.; GIMENO, S. G. A. Reprodutibilidade e validade do questionário de freqüência de consumo de alimentos. Revista de Saúde Pública, v. 36, n. 4, p. 505-512, 2002.

SHILS, M.; OLSON, J.; SHIKE, M. Tratado de nutrição moderna na saúde e na doença. São Paulo: Manole, 2003.

WHO. Safety evaluation of certain food additives and contaminants: twenty-first report of Joint FAO/WHO Expert Committee on Food Additives (JECFA). Geneva, 2000.p. 113-118. (WHO food additives series, 44). 